\title{
Effectiveness of implementing a dyadic psychoeducational intervention for cancer patients and family caregivers
}

\author{
Marita G. Titler ${ }^{1}$ - Moira A. Visovatti ${ }^{2}$ Clayton Shuman ${ }^{1}$ - Katrina R. Ellis ${ }^{3}$. \\ Tanima Banerjee $^{4} \cdot$ Bonnie Dockham $^{5,6} \cdot$ Olga Yakusheva $^{1} \cdot$ Laurel Northouse $^{7}$
}

Received: 27 January 2017 / Accepted: 16 May 2017 /Published online: 13 June 2017

(C) The Author(s) 2017. This article is an open access publication

\begin{abstract}
Purpose This study examined the effectiveness, feasibility, and satisfaction with implementation of the FOCUS program in two US Cancer Support Community affiliates in Ohio and California as well as the cost to deliver the program. FOCUS is an evidence-based psychoeducational intervention for dyads (cancer patients and caregivers).

Methods A pre-post-intervention design was employed. Eleven, five-session Focus programs were delivered by licensed professionals in a small group format (three-four dyads/group) to 36 patient-caregiver dyads. An Implementation Training Manual, a FOCUS Intervention Protocol Manual, and weekly conference calls were used to foster implementation. Participants completed questionnaires prior to and following completion of each five-session FOCUS program to measure primary (emotional distress,
\end{abstract}

Marita G. Titler

mtitler@med.umich.edu

Moira A. Visovatti

mvisovat@umich.edu

Clayton Shuman

clayshu@med.umich.edu

Katrina R. Ellis

katrina.ellis@unc.edu

Tanima Banerjee

tanima@med.umich.edu

Bonnie Dockham

bonnie@ cancersupportannarbor.org

Olga Yakusheva

yakush@med.umich.edu

Laurel Northouse

lnortho@umich.edu quality of life) and secondary outcomes (benefits of illness, self-efficacy, and dyadic communication). Enrollment and retention rates and fidelity to FOCUS were used to measure feasibility. Cost estimates were based on time and median hourly wages. Repeated analysis of variance was used to analyze the effect of FOCUS on outcomes for dyads. Descriptive statistics were used to examine feasibility, satisfaction, and cost estimates.

Results FOCUS had positive effects on QOL $(p=.014)$, emotional ( $p=.012)$, and functional $(p=.049)$ well-being, emotional distress $(p=.002)$, benefits of illness $(p=.013)$, and self-efficacy $(p=.001)$. Intervention fidelity was $85 \%$ with enrollment and retention rates of 71.4 and $90 \%$, respectively. Participants were highly satisfied. Cost for oversight and delivery of the five-session FOCUS program was $\$ 168.00$ per dyad.

1 Department of Systems, Populations and Leadership, University of Michigan School of Nursing, 400 North Ingalls Street, Room 4170, Ann Arbor, MI 48109-5482, USA

2 Department of Health Behavior and Biological Sciences, University of Michigan School of Nursing, 400 North Ingalls Street, Room 2185, Ann Arbor, MI 48109-5482, USA

3 Gillings School of Public Health, Department of Health Behavior, University of North Carolina at Chapel Hill, 302C Rosenau Hall, Chapel Hill, NC 27599-7440, USA

4 Data \& Methods Hub, North Campus Research Complex, University of Michigan Institute for Healthcare Policy \& Innovation, Building 10, Room No. A193, Ann Arbor, MI 48109, USA

5 Cancer Support Community, 2010 Hogback Road, Suite 3, Ann Arbor, MI 48105, USA

6 University of Michigan School of Social Work, 1080 South University, Ann Arbor, MI 48109, USA

7 University of Michigan School of Nursing, 400 North Ingalls Street, Room 2160, Ann Arbor, MI 48109-5482, USA 
Conclusions FOCUS is an economic and effective intervention to decrease distress and improve the quality of life for dyads.

Keywords Psychoeducational intervention · Dyadic . Cancer $\cdot$ Costs $\cdot$ Implementation $\cdot$ Caregivers

\section{Introduction}

Although cancer patients receive state-of-the-art medical care, they seldom have help coping with the detrimental effects of the illness on their quality of life (QOL) [1]. Family caregivers provide complex care in the home with little support, which reduces caregivers' self-efficacy and increases their distress [2]. Although interventions have been developed to support cancer patients and their family caregivers, they seldom move from efficacy testing to implementation in community settings where people can benefit from them [3].

The FOCUS program is an evidence-based, psychoeducational intervention that improves patient and caregiver outcomes (e.g., emotional distress, QOL) as demonstrated in three randomized clinical trials $(\underline{N}=947$ patient-caregiver pairs) [4-6]. We piloted implementation of FOCUS in one local Cancer Support Community (CSC) site with demonstrated effectiveness when implemented by agency social workers using a small group format [7]. The purpose of this study was to examine program outcomes, feasibility, and satisfaction with implementation of FOCUS in two CSC sites outside our local community. We also examined costs of delivering the FOCUS program which has not been evaluated previously.

Study aims were as follows:

1. To determine effects of the FOCUS program on patientcaregiver dyad's primary outcomes (emotional distress and quality of life) and secondary outcomes (benefits of illness, self-efficacy, dyadic communication) at CSC sites in Ohio and California

2. To assess the feasibility of program implementation at these CSC sites

3. To evaluate program satisfaction

4. To estimate costs of delivering FOCUS

Stress-coping theory $[8,9]$ is an important framework for understanding how patients and caregivers cope with cancer. According to the theory, a series of personal, social, and illness-related factors influence how patients' and caregivers' appraise the illness and cope with demands associated with it. Figure 1 illustrates the stress-coping model as it applies to the FOCUS Program and the potential intervention effects examined in this study. The program provides patients and caregivers with information and support jointly (as unit of care), which helps increase positive appraisal of the illness (i.e., benefits of illness/caregiving), enhance coping resources (i.e., self-efficacy), improve quality of life, and reduce emotional distress.

\section{Methods}

\section{Study design}

The study was approved by the University of Michigan Institutional Review Board. A pre-post-intervention design (no control group) was used. Primary and secondary outcomes were completed at baseline (time 1) prior to the intervention and again 5 weeks later (time 2), following program completion. A control group was not used because three prior randomized controlled trials demonstrated the efficacy of the FOCUS program with significant findings across studies $[4-6,10]$.

\section{Sites}

FOCUS was implemented at the CSC in Cincinnati, Ohio, and in Santa Monica, California, each serving approximately 1500 patients annually. The CSC is a large network of community agencies in the US that provides professional psychosocial care in a group format at no cost to cancer patients and their family caregivers. The racial mix at the Cincinnati CSC is principally Caucasian $(81 \%)$ and African-American $(11 \%)$ whereas the racial mix at the Santa Monica CSC site is Caucasian (69\%), Asian (11\%), and Hispanic (7\%).

\section{Participants}

Patient eligibility criteria were (1) age 18 or older; (2) diagnosis of any cancer type including advanced cancer, currently in treatment or completed treatment in the past 18 months; (3) physically and mentally able to participate as determined by the intake licensed therapist; (4) able to read and speak English; and (5) have a family caregiver willing to participate in the program. Caregiver eligibility criteria were (1) age 18 or older, (2) physically and mentally able to participate, (3) able to speak and read English, (4) identified by the patient as the primary caregiver, and (5) willing to participate in the study. Family caregiver was defined as an individual who provided emotional and/or physical support to the patient, without pay. Caregivers were excluded if they were diagnosed with cancer in the previous year or were receiving active treatment for cancer so all dyads were managing the effects of cancer in the patient, not the caregiver. A sample size of 56 dyads was planned to provide $80 \%$ power $[11,12]$ to detect a medium effect size $(d=.37)$ with alpha of .05 two tailed, based on the effects from our prior implementation study [7]. 
Fig. 1 Theoretical framework: stress-coping model
SECONDARY OUTCOMES

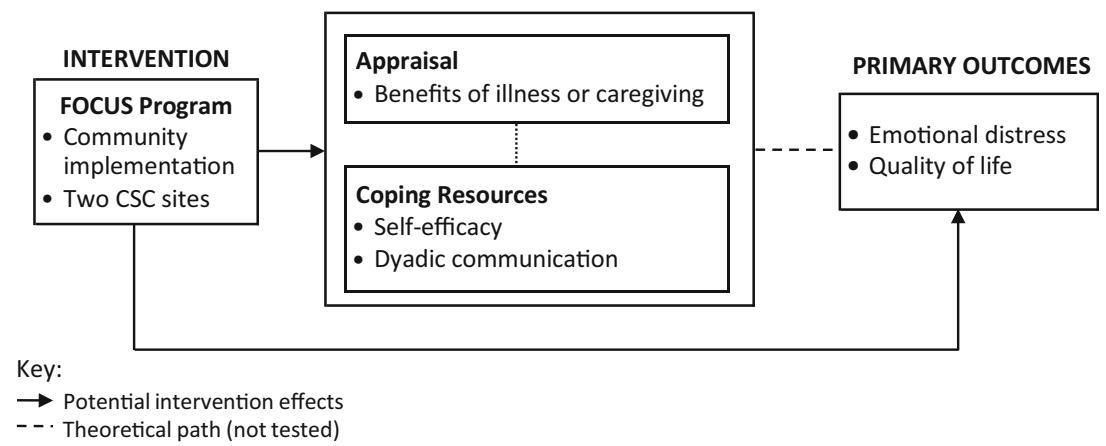

\section{The FOCUS intervention}

FOCUS is a psychoeducational intervention delivered to patient-caregiver pairs (dyads) that addresses Family involvement, Optimistic attitude, Coping effectiveness, Uncertainty reduction, and Symptom management (see Table 1) [13]. The program was delivered by a master's prepared facilitator (licensed social worker, family therapist) employed by each of the CSC sites, in a group format of three to four dyads (6-8 people), and was comprised of five weekly face-to-face sessions of 2 hours each. Eleven five-session FOCUS programs were delivered over 12 months.

\section{Implementation strategies}

Implementation was guided by the Translating Research into Practice Model in which implementation is promoted through communication among program developers and program facilitators [14-16]. Thus, an Implementation Training Manual and a FOCUS Intervention Protocol Manual were developed. The training manual was designed to educate CSC program directors and facilitators about the efficacy of FOCUS, program components, resources for implementation, and the research components (e.g., informed consent). The FOCUS Intervention Protocol Manual was designed for use by the facilitator to foster delivery and documentation of fidelity to the weekly FOCUS activities. A 1-day training program was held for CSC site program directors and facilitators (see Table 2). Other implementation strategies included (1) providing sets of pre-packaged materials to implement each 5-week FOCUS program, (2) weekly conference calls with facilitators to provide guidance on issues that arose, and (3) teleconference attendance of the facilitators at monthly investigator team meetings.

\section{Instruments}

\section{Primary outcomes}

Emotional distress was measured using the Cancer Support Source Distress Scale (CSSDS) developed by the
Cancer Support Community [17]. Reliability and validity have been demonstrated [17]. Quality of life was measured using the Functional Assessment of Cancer Treatment-General (FACT-G) QOL Scale (version 4) [18]. The FACT-G has demonstrated validity, and both the original and modified versions have demonstrated reliability in cancer patients and their caregivers [5-7] (see Table 3).

\section{Secondary outcomes}

The Benefits of Illness Scale [20] was used to measure patients' and caregivers' perceived benefits arising from the cancer illness (e.g., led me to be more accepting of things). Validity and reliability have been demonstrated [20]. The Lewis' Cancer Self-efficacy Scale (CASE) was used to measure confidence to manage cancer [21]. Validity and reliability have been demonstrated in multiple studies [5, 6, 21]. The Lewis' Mutuality and Interpersonal Sensitivity Scale (MIS) was used to measure communication between the patient and caregiver about cancer [21]. Validity and reliability have been demonstrated in multiple studies $[6,7,21]$ (see Table 3). Demographic questions assessed participants' age, gender, marital status, race, education, income, and employment status. Medical-related questions assessed patients' type and stage of cancer, and whether they were currently receiving treatment.

\section{Feasibility}

Feasibility was measured by (1) enrollment and retention rates and (2) intervention fidelity. Data regarding fidelity to the FOCUS program was collected through the fidelity checklist in the FOCUS protocol manual, completed by the facilitator, for activities to be done during each of the five sessions. Activities to complete across the five sessions were 104 (session $1=25$; session $2=17$; session $3=18$; session $4=19$; session $5=25$ ). 


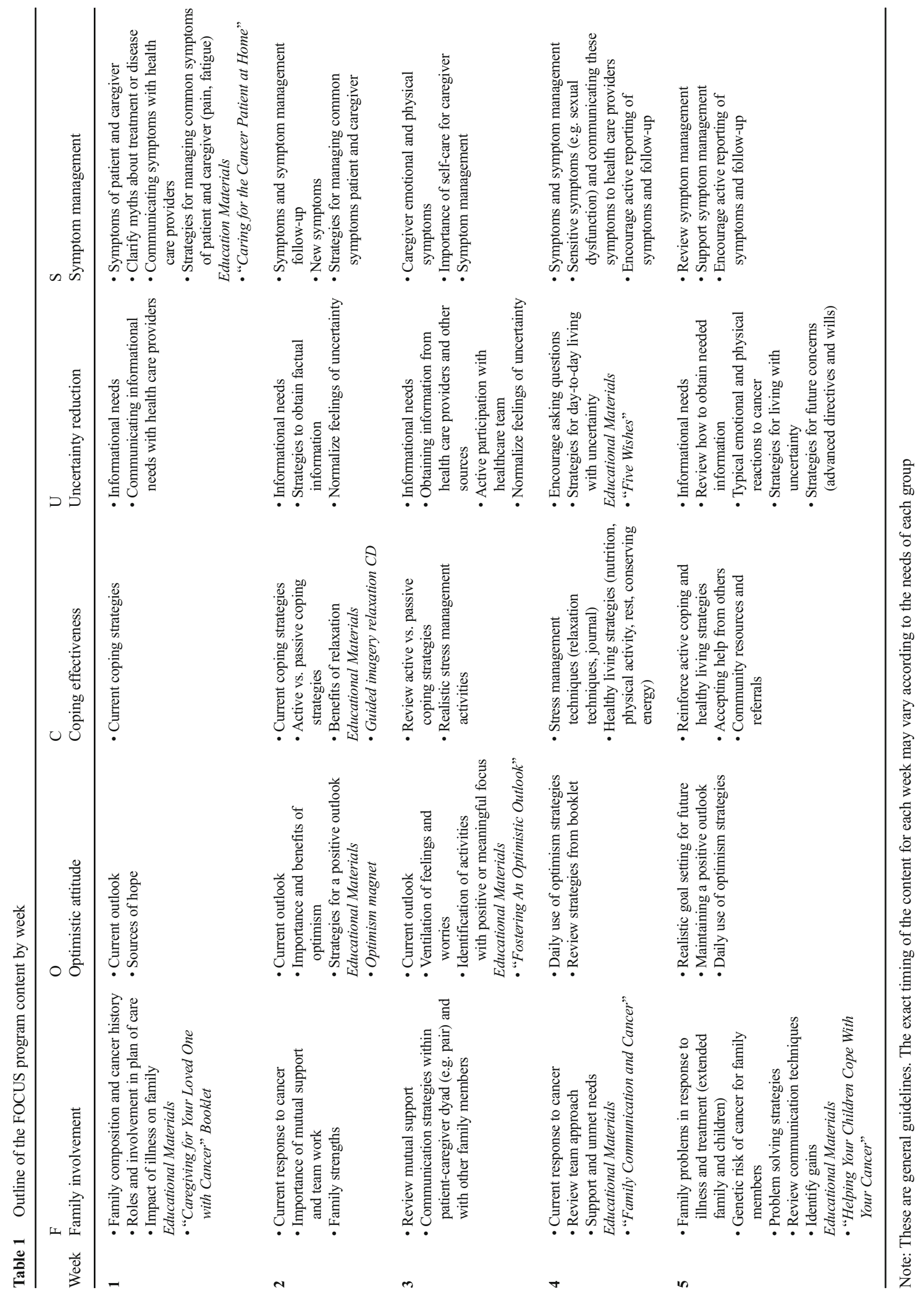


Table 2 Outline of the training program for CSC sites

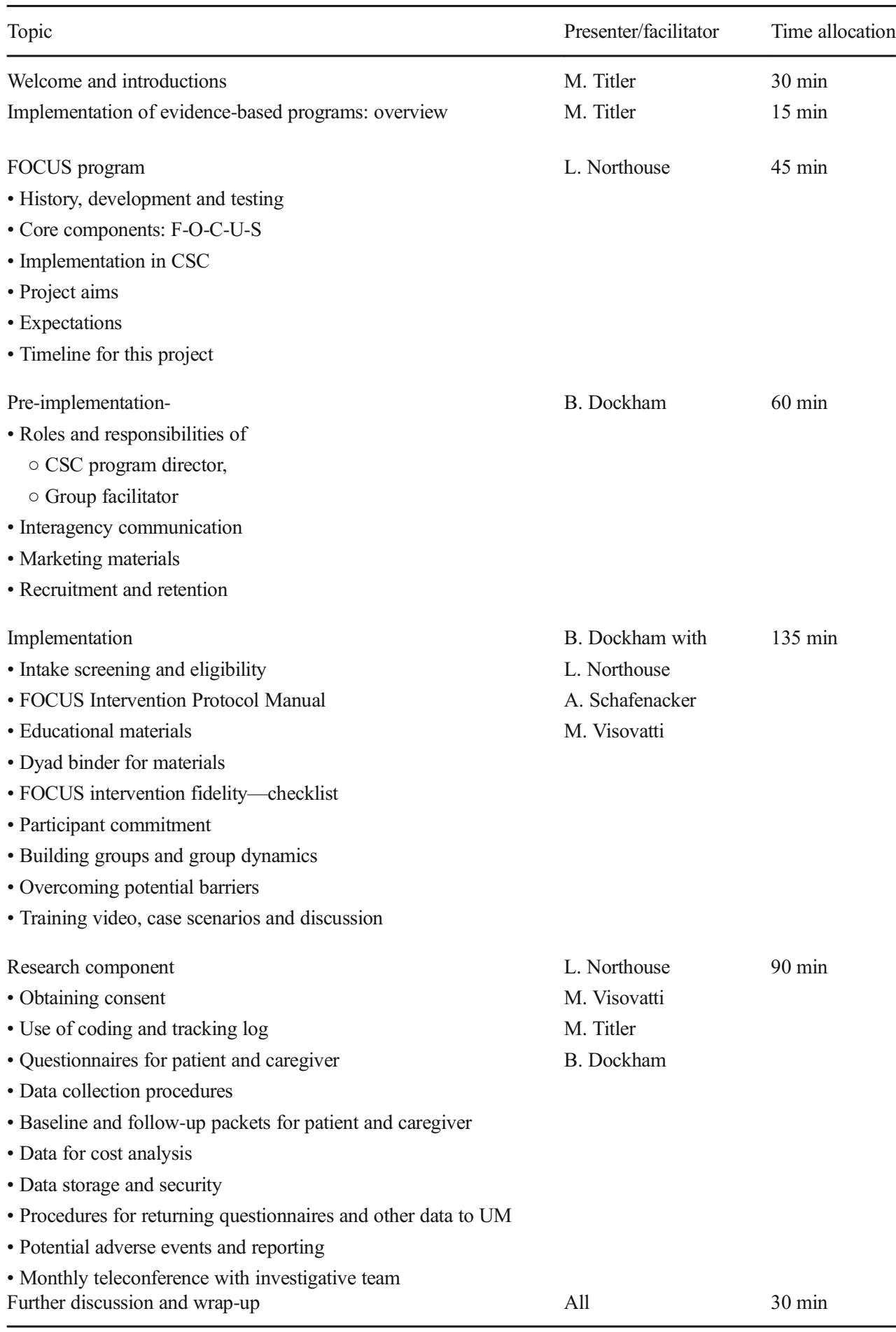

\section{Satisfaction}

Satisfaction was measured by the FOCUS Satisfaction Instrument [7]. It includes seven items for rating satisfaction with components of the program $(1=$ not satisfied to $5=$ very satisfied); ratings are summed and divided by seven to arrive at overall satisfaction scores. It includes open-ended questions regarding session lengths and FOCUS program length.

\section{Program costs}

Program costs were calculated by multiplying hourly time estimates and median hourly wages for healthcare social workers in California and Ohio according to the US Bureau of Labor Statistics, May 2015 (CA: \$32.53; OH: \$26.01). Costs were estimated for the average total cost to deliver one five-session FOCUS program at each site and across sites. 
Table 3 Primary and secondary outcome measures

\begin{tabular}{|c|c|c|}
\hline Instrument name & Description & Scoring and interpretation \\
\hline $\begin{array}{l}\text { Cancer Support Source } \\
\text { Distress Scale [17] }\end{array}$ & $\begin{array}{l}\text { Consists of } 15 \text { items to measure the extent of concern } \\
(0=\text { not at all to } 5=\text { very seriously }) \text { about various } \\
\text { emotions related to the cancer experience }\end{array}$ & $\begin{array}{l}\text { Score of } 0 \text { to } 60 \text { is calculated by summing ratings } \\
\text { across all items. Higher scores indicate greater } \\
\text { emotional distress }\end{array}$ \\
\hline FACT-G (version 4) [18] & $\begin{array}{l}\text { Consists of } 27 \text { items to measure overall QOL, and the } \\
\text { domains for physical, social, emotional, and functional } \\
\text { well-being in cancer patients }(0=\text { not at all to } 4=\text { very } \\
\text { much). For caregivers, FACT-G was slightly modified } \\
\text { using nearly identical items [19] }\end{array}$ & $\begin{array}{l}\text { QOL score of } 0-108 \text { is calculated by summing } \\
\text { item ratings across all domains. Domain scores } \\
\text { are calculated by summing individual items } \\
\text { within the domain. Higher scores indicate higher } \\
\text { well-being (subscales) and QOL }\end{array}$ \\
\hline Benefits of Illness Scale [20] & $\begin{array}{l}\text { Consists of } 11 \text { items to measure patients and caregivers } \\
\text { perceived benefits }(1=\text { not at all to } 4=\text { a lot }) \text { arising } \\
\text { from the cancer illness }\end{array}$ & $\begin{array}{l}\text { Score of } 1 \text { to } 4 \text { is calculated by summing ratings on } \\
\text { all items and dividing by } 11 \text {. Higher scores } \\
\text { indicate more perceived benefits of illness }\end{array}$ \\
\hline Lewis' Cancer Self-efficacy Scale [21] & $\begin{array}{l}\text { Consists of } 17 \text { items to measure patients' and caregivers } \\
\text { confidence to manage cancer }(0=\text { not at all to } \\
10=\text { very confident })\end{array}$ & $\begin{array}{l}\text { Score of } 0 \text { to } 170 \text { is calculated by summing ratings } \\
\text { across all items. Higher scores reflect higher } \\
\text { self-efficacy }\end{array}$ \\
\hline $\begin{array}{l}\text { Lewis' Mutuality and Interpersonal } \\
\text { Sensitivity Scale [21] }\end{array}$ & $\begin{array}{l}\text { Consists of } 23 \text { items to measure dyadic communication } \\
\text { about cancer }(1=\text { never true to } 5=\text { always true })\end{array}$ & $\begin{array}{l}\text { Score of } 1 \text { to } 5 \text { is calculated by summing ratings } \\
\text { across all items and dividing by } 23 \text {. Higher } \\
\text { scores indicate more perceived open } \\
\text { communication between the patient and } \\
\text { caregiver about cancer }\end{array}$ \\
\hline
\end{tabular}

Time estimates Preparation and wrap-up time for each of the five sessions were estimated as $20 \mathrm{~min}$ based on our previous study [7]. Facilitators at each of the two CSC sites logged the session time in minutes at the end of each session on the fidelity checklist. We summed across all 11 FOCUS Programs, (1) the logged session times and (2) the preparation and wrap-up times to arrive at the total time to deliver FOCUS; this was then divided by 11 (number of programs delivered) to arrive at the mean time to deliver a five-session FOCUS program. The mean total time across both sites to deliver FOCUS including preparation and wrap-up was $11.2 \mathrm{~h}(\mathrm{SD}=0.3)$. We also accounted for estimated time for program oversight. These estimates per FOCUS program are based on our prior study as follows [7]: marketing - $1.5 \mathrm{~h}$; recruiting and outreach $-1 \mathrm{~h}$; intake and screening for eligibility $-7 \mathrm{~h}$; and organization of education materials $-1.5 \mathrm{~h}$. These oversight estimates ( $11 \mathrm{~h}$ ) were added to the mean time to deliver a five-session FOCUS program (11.2 h), as described above, resulting in an estimated $22.2 \mathrm{~h}$ to provide oversight and delivery of one five-session FOCUS program.

Cost estimates To calculate cost estimates, the time to provide oversight and delivery of FOCUS (22.2 h) was multiplied by the median hourly wage for each respective site, resulting in site specific cost estimates. The average cost across sites to provide oversight and deliver one FOCUS program was calculated by multiplying the site specific costs by the number of FOCUS programs delivered at each site. These values were then summed and divided by 11, the number of FOCUS programs delivered across sites.

\section{Data collection procedures}

Patient and caregiver versions of the baseline (time 1) questionnaires consisting of Cancer Support Source Distress Scale, FACT-G QOL Scale, Benefits of Illness Scale, Lewis' Cancer Self-Efficacy Scale, Lewis' Mutuality and Interpersonal Sensitivity Scale (see Table 3), and demographic and medical-related questions were completed prior to beginning the FOCUS program. Questionnaires were mailed or given to participants during the intake visit and collected immediately prior to the first FOCUS program session. Patients and caregivers completed questionnaires independently. Follow-up questionnaires (time 2) included the major scales, noted above, and the Focus Satisfaction Instrument, and were administered immediately after the last FOCUS session.

\section{Data analysis}

For aim 1, repeated measures analysis of variance was used to determine if changes occurred in primary and secondary outcomes from time 1 to time 2 for patients and caregivers as dyads (i.e., pairs). Dyadic analysis was used because patients' and caregivers' responses to illness are interrelated [22, 23]. Time and role (patient vs. caregiver) were treated as within-subject variables to control for the interdependent nature of the data. Main effect by time was examined to determine the overall effectiveness of the FOCUS intervention on primary and secondary outcomes for patientcaregiver dyads as a unit. Time-by-role interactions were analyzed to determine if there was a differential effect of the intervention on patients' and caregivers' outcomes. 
Cancer stage was used as a control variable in the main effect analysis (effect of intervention on outcome) and interaction effect analysis (differential effect of intervention on patients' and caregivers' outcomes) for physical and functional well-being because significant associations $(p<0.05)$ were found between cancer stage and these outcomes for patients ( $F=3.46$ for physical; $F=2.83$ for functional). Intervention effect sizes were examined for specific variables based on conventional standards $($ small $=0.20 ;$ medium $=0.50 ;$ large $=0.80)[11]$.

Descriptive statistics were used to examine enrollment and retention rates and intervention fidelity (aim 2). The enrollment rate was calculated by the number of dyads enrolled in the FOCUS program divided by the number eligible to participate. The retention rate was calculated by the number of dyads who completed the FOCUS program divided by the number who enrolled. For intervention fidelity, there were 104 total activities to complete across the five sessions per FOCUS program, and a total of 11 FOCUS programs were delivered. Fidelity for the FOCUS program was calculated by summing the number of activities documented as provided across all sessions for the 11 FOCUS programs delivered divided by total activities possible by programs $(104 \times 11)$. This resulted in a mean fidelity for the FOCUS program which had been delivered 11 times.

Descriptive statistics were used to examine patients' and caregivers' total satisfaction scores (aim 3) with follow-up exploratory analyses using paired $t$ tests to examine differences in patients' and caregivers' scores. Calculation of estimated program costs is described above.

\section{Results}

\section{Participant characteristics}

The final sample was 36 dyads (see Fig. 2). Patients' mean age was 60.8 and caregivers' was 55.9 (see Table 4). The majority of participants were female (patients, 52.8; caregivers, 55.6), and most were married or partnered $(>85 \%)$. The sample was largely white, with a moderate income. Most were well educated with a college education or higher. Most patients had stage II or IV cancer and were currently in treatment (69.4\%).

\section{Effectiveness of the FOCUS program intervention}

The FOCUS program was effective (see Table 5). The intervention had significant positive effects on dyads' primary outcomes of total QOL $(p=.014)$, emotional $(p=.012)$ and functional $(p=.049)$ well-being, and emotional distress $(p=.002)$. The intervention did not have significant effects on physical and social well-being. The intervention had significant positive effects on the dyads' secondary outcomes of benefits of illness $(p=.013)$ and self-efficacy $(p=.001)$ but not on dyadic communication regarding cancer. Reliability of instruments in this study was high (see Table 5).
Fig. 2 FOCUS CSC consort diagram final $N=36$ dyads

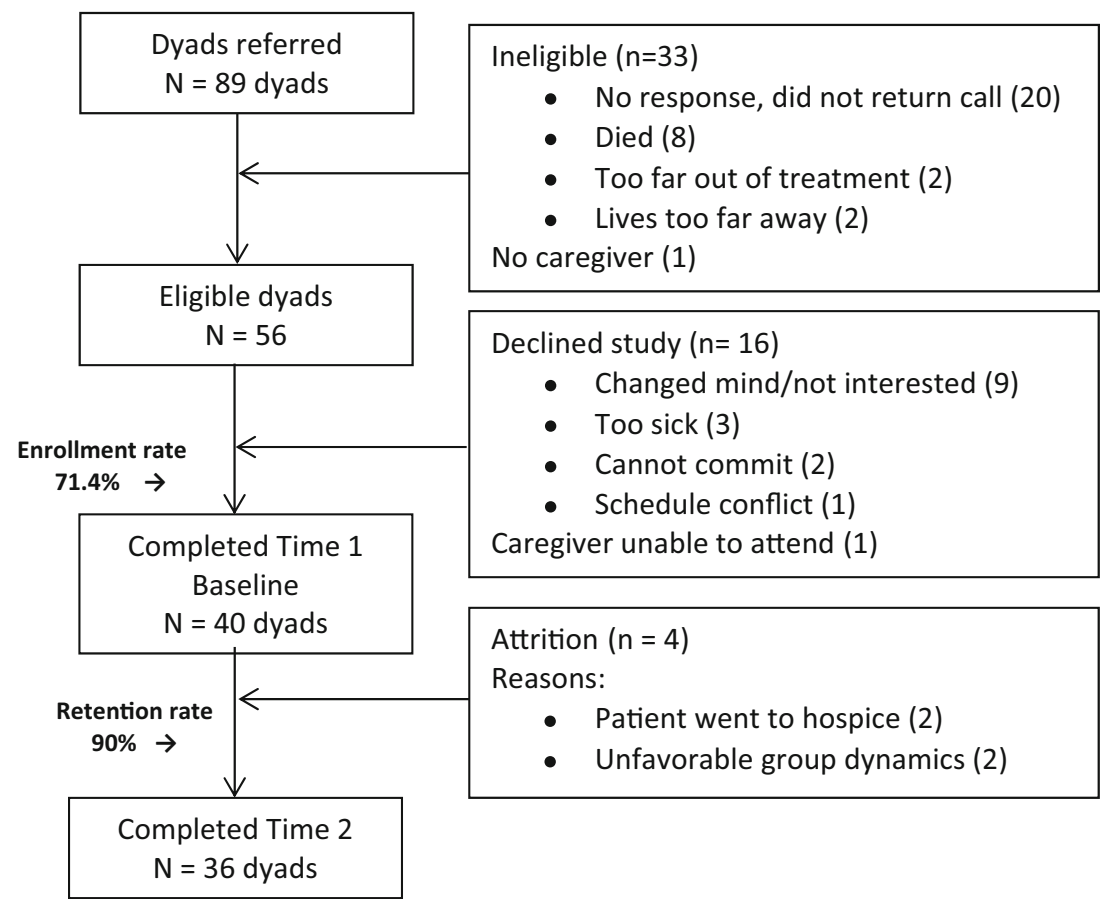


Table 4 Demographic and medical characteristics of sample

\begin{tabular}{|c|c|c|c|c|c|c|c|}
\hline \multirow[t]{2}{*}{ Characteristics } & & \multicolumn{3}{|c|}{ Patients $(n=36)$} & \multicolumn{3}{|c|}{ Caregivers $(n=36)$} \\
\hline & & Mean & $\mathrm{SD}$ & Range & Mean & $\mathrm{SD}$ & Range \\
\hline \multirow[t]{2}{*}{ Age } & & 60.8 & 14.2 & $18-88$ & 55.9 & 15.1 & $19-83$ \\
\hline & & $n$ & $\%$ & & $n$ & $\%$ & \\
\hline \multirow[t]{2}{*}{ Gender } & Male & 17 & 47.2 & & 16 & 44.4 & \\
\hline & Female & 19 & 52.8 & & 20 & 55.6 & \\
\hline \multirow[t]{3}{*}{ Marital status } & Married/partnered & 32 & 88.9 & & 31 & 86.1 & \\
\hline & Single & 3 & 8.3 & & 5 & 13.9 & \\
\hline & Divorced & 1 & 2.8 & & & & \\
\hline \multirow[t]{6}{*}{ Race } & White & 32 & 88.9 & & 28 & 77.8 & \\
\hline & Asian & 1 & 2.8 & & 3 & 8.3 & \\
\hline & Black or African-American & 1 & 2.8 & & 1 & 2.8 & \\
\hline & Latino or Hispanic & 1 & 2.8 & & 1 & 2.8 & \\
\hline & Other & 1 & 2.8 & & 1 & 2.8 & \\
\hline & Missing & & & & 2 & 5.5 & \\
\hline \multirow[t]{7}{*}{ Education } & Less than high school & 2 & 5.6 & & & & \\
\hline & High school grad or GED & 1 & 2.8 & & 1 & 2.8 & \\
\hline & Some college, technical or vocational school & 6 & 16.7 & & 7 & 19.4 & \\
\hline & College graduate & 7 & 19.4 & & 14 & 38.9 & \\
\hline & Some graduate school & 3 & 8.3 & & 1 & 2.8 & \\
\hline & Graduate degree & 17 & 47.2 & & 12 & 33.3 & \\
\hline & Prefer not to share & & & & 1 & 2.8 & \\
\hline \multirow[t]{4}{*}{ Income } & Less than $\$ 39,999$ & 3 & 8.3 & & 1 & 2.8 & \\
\hline & $\$ 40,000-\$ 99,999$ & 14 & 38.9 & & 14 & 38.9 & \\
\hline & $\$ 100,000$ or above & 16 & 44.4 & & 15 & 41.7 & \\
\hline & Missing & 3 & 8.3 & & 5 & 13.9 & \\
\hline \multirow[t]{7}{*}{ Employment } & Full time & 5 & 13.9 & & 17 & 47.2 & \\
\hline & Part time & 5 & 13.9 & & 2 & 5.6 & \\
\hline & Not employed & 9 & 25.0 & & 5 & 13.9 & \\
\hline & Retired & 13 & 36.1 & & 9 & 25.0 & \\
\hline & Disabled & 4 & 11.1 & & & & \\
\hline & Student & & & & 1 & 2.8 & \\
\hline & Missing & & & & 2 & 5.6 & \\
\hline \multirow[t]{12}{*}{ Type of cancer } & Breast & 6 & 16.7 & & & & \\
\hline & Pancreatic & 4 & 11.1 & & & & \\
\hline & Gynecologic & 4 & 11.1 & & & & \\
\hline & Prostate & 4 & 11.1 & & & & \\
\hline & Brain & 3 & 8.3 & & & & \\
\hline & Lung & 3 & 8.3 & & & & \\
\hline & Lymphoma & 3 & 8.3 & & & & \\
\hline & Multiple myeloma & 2 & 5.6 & & & & \\
\hline & Colorectal/GI & 2 & 5.6 & & & & \\
\hline & Kidney/adrenal & 2 & 5.6 & & & & \\
\hline & Nose/throat & 2 & 5.6 & & & & \\
\hline & Skin (non-melanoma) & 1 & 2.8 & & & & \\
\hline \multirow[t]{6}{*}{ Stage of cancer } & I & 6 & 16.7 & & & & \\
\hline & II & 8 & 22.2 & & & & \\
\hline & III & 6 & 16.7 & & & & \\
\hline & IV & 13 & 36.1 & & & & \\
\hline & Unknown & 2 & 5.6 & & & & \\
\hline & Missing & 1 & 2.8 & & & & \\
\hline \multirow[t]{3}{*}{ Currently in treatment } & Yes & 25 & 69.4 & & & & \\
\hline & No & 10 & 27.8 & & & & \\
\hline & Missing & 1 & 2.8 & & & & \\
\hline
\end{tabular}


Table 5 Effectiveness of the FOCUS program intervention $(N=36$ dyads $)$

\begin{tabular}{|c|c|c|c|c|c|c|c|c|c|c|}
\hline \multirow[b]{3}{*}{ Outcomes } & \multirow[b]{3}{*}{ Reliabilities $^{\mathrm{a}}$} & \multicolumn{4}{|l|}{ Mean (SD) } & \multicolumn{2}{|l|}{$p$ values } & \multicolumn{3}{|c|}{ Effect sizes $^{b}$} \\
\hline & & \multicolumn{2}{|l|}{ Patient } & \multicolumn{2}{|c|}{ Caregiver } & \multirow{2}{*}{$\begin{array}{l}\text { Main effect } \\
\text { Time* }\end{array}$} & \multirow{2}{*}{$\begin{array}{l}\text { Interaction effect } \\
\text { Role by time** }\end{array}$} & \multirow{2}{*}{$\begin{array}{l}\text { Patient } \\
d\end{array}$} & \multirow{2}{*}{$\begin{array}{l}\text { Caregiver } \\
d\end{array}$} & \multirow{2}{*}{$\begin{array}{l}\text { Dyad } \\
d\end{array}$} \\
\hline & & Time 1 & Time 2 & Time 1 & Time 2 & & & & & \\
\hline \multicolumn{11}{|l|}{ Quality of life } \\
\hline Total QOL & $.92 ; .86$ & $\begin{array}{l}67.24 \\
(17.40)\end{array}$ & $\begin{array}{l}71.52 \\
(16.91)\end{array}$ & $\begin{array}{l}72.59 \\
(11.97)\end{array}$ & $\begin{array}{l}76.11 \\
(15.72)\end{array}$ & .014 & .826 & 0.249 & 0.252 & 0.309 \\
\hline Physical & $.84 ; .73$ & $\begin{array}{l}17.67 \\
(5.47)\end{array}$ & $\begin{array}{l}18.58 \\
(6.38)\end{array}$ & $\begin{array}{l}24.29 \\
(2.72)\end{array}$ & $\begin{array}{l}23.75 \\
(4.86)\end{array}$ & .986 & .412 & 0.153 & 0.137 & 0.030 \\
\hline Social & $.84 ; .71$ & $\begin{array}{l}18.15 \\
(5.85)\end{array}$ & $\begin{array}{l}19.51 \\
(5.37)\end{array}$ & $\begin{array}{l}18.08 \\
(4.98)\end{array}$ & $\begin{array}{l}18.77 \\
(5.11)\end{array}$ & .061 & .392 & 0.242 & 0.136 & 0.214 \\
\hline Emotional & $.86 ; .83$ & $\begin{array}{l}14.92 \\
(5.97)\end{array}$ & $\begin{array}{l}16.42 \\
(5.04)\end{array}$ & $\begin{array}{l}13.53 \\
(4.40)\end{array}$ & $\begin{array}{l}14.64 \\
(5.59)\end{array}$ & .012 & .695 & 0.272 & 0.221 & 0.341 \\
\hline Functional & $.79 ; .84$ & $\begin{array}{l}16.51 \\
(4.89)\end{array}$ & $\begin{array}{l}17.01 \\
(5.15)\end{array}$ & $\begin{array}{l}16.57 \\
(4.59)\end{array}$ & $\begin{array}{l}18.95 \\
(5.34)\end{array}$ & .049 & .520 & 0.010 & 0.477 & 0.400 \\
\hline Benefits of illness & $.88 ; .92$ & $\begin{array}{l}2.88 \\
(.65)\end{array}$ & $\begin{array}{l}2.97 \\
(.61)\end{array}$ & $\begin{array}{l}2.80 \\
(.65)\end{array}$ & $\begin{array}{l}2.98 \\
(.77)\end{array}$ & .013 & .731 & 0.143 & 0.253 & 0.264 \\
\hline Communication & $.96 ; .96$ & $\begin{array}{l}3.52 \\
(.91)\end{array}$ & $\begin{array}{l}3.62 \\
(.75)\end{array}$ & $\begin{array}{l}3.49 \\
(.80)\end{array}$ & $\begin{array}{l}3.64 \\
(.77)\end{array}$ & .075 & .321 & 0.120 & 0.191 & 0.186 \\
\hline Emotional distress & $.89 ; .90$ & $22.61(11.67)$ & $\begin{array}{l}18.31 \\
(9.38)\end{array}$ & $\begin{array}{l}20.86 \\
(9.59)\end{array}$ & $\begin{array}{l}18.58 \\
(10.58)\end{array}$ & .002 & .236 & 0.406 & 0.226 & 0.411 \\
\hline Self-efficacy & $.96 ; .97$ & $113.97(32.43)$ & $\begin{array}{l}119.11 \\
(29.35)\end{array}$ & $\begin{array}{l}105.08 \\
(32.31)\end{array}$ & $\begin{array}{l}122.47 \\
(32.23)\end{array}$ & .001 & .052 & 0.166 & 0.517 & 0.512 \\
\hline
\end{tabular}

${ }^{a}$ Reliabilities are the average across time points for patients and caregivers

${ }^{\mathrm{b}}$ Absolute value of effect size (Cohen's $\left.d\right) d=($ mean 1 - mean 2$) / s$ where $s=$ pooled standard deviation

*Significance ( $p$ value) of main effect by time: does the outcome change from time 1 to time 2 for the dyad; significant findings $(p<.05)$ are italicized **Significance ( $p$ value) of interaction effect of role by time: does the amount of change from time 1 to time 2 differ for patients and caregivers; no significant findings $(p>.05)$

\section{Feasibility}

As displayed in Fig. 2, the enrollment rate was $71.4 \%$ and the retention rate was $90 \%$. Intervention fidelity was $85 \%$.

\section{Satisfaction}

Caregivers and patients reported high satisfaction, and scores did not differ significantly [(patients: 4.4 (SD 0.6); caregivers: 4.5 (SD 0.5$)]$. The majority of patients $(86.1 \%)$ and caregivers $(80.6 \%)$ reported the program helped them cope with cancer. Most reported that the program did not duplicate services provided at their treatment center $(85.3 \%$ each). When asked about the length of each session (about right; shorter; longer), the majority of patients $(86.1 \%)$ and caregivers $(80.6 \%)$ noted the session lengths were about right. When asked about the number of group sessions (about right, fewer, more), $52.8 \%$ of the patients and $50 \%$ of the caregivers wanted more; $47.2 \%$ noted the number was about right. Over $90 \%$ of patients and caregivers noted they would recommend the program to others facing cancer.

\section{Estimated program costs}

Due to differing geographic hourly wage rates, California estimated costs $(\$ 722.17)$ were higher than Ohio $(\$ 577.42)$. Average cost estimates for oversight and delivery of one five-session FOCUS program was $\$ 669.45$ or $\$ 168.00$ per dyad, assuming four dyads per group.

\section{Discussion}

\section{Program effectiveness}

The FOCUS program was effective in improving the primary outcomes of quality of life, emotional and functional wellbeing, and emotional distress. This is consistent with other couple-based group interventions for cancer patients and their 
partners [24]. In qualitative statements, patients and caregivers reported that it was very helpful to have their partner or caregiver attend the group intervention with them. Both patients and caregivers were able to express feelings and learn from others, and this opportunity may have helped improve their QOL and emotional distress. FOCUS also was effective in improving perceived benefits of experiencing cancer (benefits of illness) and level of confidence in managing it (self-efficacy). Prior research with cancer patients and caregivers has demonstrated that group interventions facilitate positive reappraisal of the illness [25] and can increase caregivers' preparedness and competence to provide care [26]. The findings and effect sizes obtained for the domains of QOL, benefits of illness, and self-efficacy in this study are similar to those obtained in our prior randomized trials when FOCUS was delivered by a nurse to individual dyads at home [4-6, 27] and in our previous implementation study [7]. The magnitude of the intervention effects in this study are similar to small-medium effect sizes reported in three meta-analyses examining intervention effects with patients and caregivers across multiple studies [3, 28, 29].

Participants' physical well-being, social well-being, and dyadic communication did not improve. The lack of improvement in physical well-being (e.g., nausea, pain) is not surprising because FOCUS is a psychoeducational intervention and improvements in physical well-being require tailored interventions that address the biological components of cancer. However, there was an improvement in functional wellbeing (e.g., ability to work; enjoy life). Perhaps, participants learned how to address physical challenges and related symptoms so that they could function in their daily lives. The lack of improvement in social well-being may be due to higher baseline social well-being scores with less room for improvement. Lack of improvement in dyadic communication is difficult to explain. FOCUS may have stimulated beginning communication about cancer that did not carry over into participants' daily lives. Additional work regarding how communication is conceptualized and measured as an outcome of FOCUS may be warranted in future studies (i.e., qualitative approach with participants).

\section{Feasibility}

It is feasible to deliver FOCUS in a group format in CSC sites. Enrollment rates were good (71.4\%) and higher than those reported in other studies using group interventions with patient-caregiver dyads (10.4\%) [24] or family caregivers $(25-31 \%)[30,31]$. Perhaps this is because agency staff recruited participants and delivered the intervention. Retention rates $(90 \%)$ were high most likely because of perceived benefits from the group and its dyadic approach.

FOCUS was delivered with high intervention fidelity $(85 \%)$ and was similar to a couple-focused group intervention with breast cancer patients (88\%) [24]. The implementation strategies were important for providing initial and ongoing support to the group facilitators and helped maintain high intervention fidelity.

\section{Satisfaction}

Participants were highly satisfied with the FOCUS program and the overwhelming majority would recommend the program to others. About half of the participants wanted more sessions, raising the issue of optimal intervention dose. In our prior implementation study, using a six-session group intervention, $>40 \%$ of the participants also reported they would have liked more sessions [7]. One challenge in planning an intervention is balance between feasibility (i.e., enrollment, retention) and outcomes (i.e., program effectiveness). Manne et al. [24] found a low enrollment rate (10.4\%) among cancer patients who were approached for an 8-week group intervention, with $15.7 \%$ expressing worry about the program length. Bultz et al. [30] found low enrollment (31\%) for a 6-week group intervention for partners, with $29 \%$ stating time was an issue. Determining optimal intervention dose needs further exploration.

\section{Program cost}

This study provided the first cost estimate of delivering the five-session FOCUS program; about $\$ 168.00$ per dyad. Badger et al. [32] estimated costs of their 8-week dyadic telephone intervention as $\$ 164.68$ per dyad but omitted oversight and preparation costs included in our estimates. Given the skyrocketing healthcare costs in the US, FOCUS is highly economical to address critical issues of those living with cancer.

\section{Limitations}

One limitation is enrollment of 36 rather than 56 dyads as planned. Despite this limitation, the dyadic effects for many variables were $>0.30$, and we were able to demonstrate the effectiveness of the FOCUS program. Performing costeffective analyses was not a study aim and requires use of a control group which deprives patients and caregivers from an intervention that has demonstrated efficacy and effectiveness. We did, however, provide a cost estimate for delivery of FOCUS in CSCs.

\section{Conclusions}

FOCUS delivered in a group, dyadic format is an economically feasible intervention that decreases emotional distress and improves quality of life, perceived benefits from 
experiencing cancer, and confidence in managing it. It is time that programs like FOCUS receive national public policy attention to effect payment structures for delivery to cancer patients and their caregivers.

Acknowledgments The authors would like to acknowledge the important contributions of (1) William Kavanaugh, MACP, LMFT, Program Manager, Cancer Support Community-Benjamin Center, Los Angeles, California, and (2) Kelly Schoen, LISW-S, Program Director, Cancer Support Community Greater Cincinnati-Northern Kentucky, Blue Ash, Ohio, who were instrumental in recruitment and program delivery.

\section{Compliance with ethical standards}

Conflict of interest The authors declare that they have no competing interests.

\section{Research involving human participants and/or animals}

1. Statement of human rights: All procedures performed in studies involving human participants were in accordance with the ethical standards of the institutional and/or national research committee and with the 1964 Helsinki declaration and its later amendments or comparable ethical standards.

2. Statement of welfare of animals. This article does not contain any studies with animals performed by any of the authors.

Informed consent Informed consent was obtained from all participants included in the study.

Funding source This study was funded by the Michigan Institute for Clinical and Health Research Pilot Grant Program, University of Michigan, UL1TR000433.

Open Access This article is distributed under the terms of the Creative Commons Attribution-NonCommercial 4.0 International License (http:// creativecommons.org/licenses/by-nc/4.0/), which permits any noncommercial use, distribution, and reproduction in any medium, provided you give appropriate credit to the original author(s) and the source, provide a link to the Creative Commons license, and indicate if changes were made.

\section{References}

1. Institute of Medicine and National Research Council (2006) From cancer patient to cancer survivor: lost in transition. National Academies Press, Washington, D.C. doi:10.17226/11468

2. van Ryn M, Sanders S, Kahn K, van Houtven C, Griffin JM, Martin M, Atienza AA, Phelan S, Finstad D, Rowland J (2011) Objective burden, resources, and other stressors among informal caregivers: a hidden quality issue? Psychooncology 20:44-52. doi:10.1002/pon. 1703

3. Northouse LL, Katapodi MC, Song L, Zhang L, Mood DW (2010) Interventions with family caregivers of cancer patients: metaanalysis of randomized trials. CA Cancer J Clin 60:317-339. doi: $10.3322 /$ caac. 20081
4. Northouse LL, Kershaw T, Mood DW, Schafenacker A (2005) Effects of a family intervention on the quality of life of women with recurrent breast cancer and their family caregivers. Psychooncology 14:478-491. doi:10.1002/pon.871

5. Northouse LL, Mood DW, Schafenacker A, Kalemkerian G, Zalupski M, LoRusso P, Hayes DF, Hussain M, Ruckdeschel J, Fendrick AM, Trask PC, Ronis DL, Kershaw T (2013) Randomized clinical trial of a brief and extensive dyadic intervention for advanced cancer patients and their family caregivers. Psychooncology 22:555-563. doi:10.1002/pon.3036

6. Northouse LL, Mood DW, Schafenacker A, Montie JE, Sandler HM, Forman JD, Hussain M, Pienta KJ, Smith DC, Kershaw T (2007) Randomized clinical trial of a family intervention for prostate cancer patients and their spouses. Cancer 110:2809-2818. doi: 10.1002/cncr.23114

7. Dockham B, Schafenacker A, Yoon H, Ronis DL, Kershaw T, Titler MG, Northouse LL (2016) Implementation of a psychoeducational program for cancer survivors and family caregivers at a cancer support community affiliate: a pilot effectiveness study. Cancer Nurs 39:169-180. doi:10.1097/NCC.0000000000000311

8. Lazarus RS (2000) Evolution of a model of stress, coping, and discrete emotions. In: Rice VH (ed) Handbook of stress, coping, and health. Sage, Thousand Oaks, pp 195-222

9. Lazarus RS, Folkman S (1984) Stress, appraisal, and coping. Springer, New York

10. Northouse LL, Schafenacker A, Barr KLC, Katapodi M, Yoon H, Brittain K, Song L, Ronis DL, An L (2014) A tailored web-based psychoeducational intervention for cancer patients and their family caregivers. Cancer Nurs 37:321-330. doi:10.1097/NCC. 0000000000000159

11. Cohen J (1988) Statistical power analysis for the behavioral sciences, 2nd edn. Lawrence Erlbaum, Hillsdale

12. Hintze JL (2000) PASS 2000 user's guide. Number Cruncher Statistical Systems, Kaysville

13. Northouse LL, Walker J, Schafenacker A, Mood D, Mellon S, Galvin E, Harden J, Freeman-Gibb L (2002) A family-based program of care for women with recurrent breast cancer and their family members. Oncol Nurs Forum 29:1411-1419. doi:10.1188/ 02.ONF.1411-1419

14. Rogers EM (2003) Diffusion of innovations. The Free Press, New York

15. Titler MG (2010) Translation science and context. Res Theory Nurs Pract 24:35-55. doi:10.1891/1541-6577.24.1.35

16. Titler MG, Herr K, Brooks JM, Xie X-J, Ardery G, Schilling ML, Marsh JL, Everett LQ, Clarke WR (2009) Translating research into practice intervention improves management of acute pain in older hip fracture patients. Health Serv Res 44:264-287. doi:10.1111/j. 1475-6773.2008.00913.x

17. Buzaglo J, Miller MF, Gayer C, Morris A, Kennedy V, Golant M (2014) CancerSupportSource: validating a 13-item web-based distress screening tool in the community. National Comprehensive Cancer Network $19^{\text {th }}$ Annual Conference, March 13-15, 2014, Hollywood, FL. http://www.cancersupportcommunity.org/sites/ default/files/uploads/our-research/presentations/distress-screeningreferral/nccn css-13 validation poster.pdf. Accessed 25 Jan 2017

18. Cella DF, Tulsky DS, Gray G, Sarafian B, Linn E, Bonomi A, Silberman M, Yellen SB, Winicour P, Brannon J, Eckberg K, Lloyd S, Purl S, Blendowski C, Goodman M, Barnicle M, Stewart I, McHale M, Bonomi P, Kaplan E, Taylor S IV, Thomas CR, Harris J (1993) The functional assessment of cancer therapy scale: development and validation of the general measure. J Clin Oncol 11:570-579. doi:10.1200/JCO. 1993.11.3.570

19. Northouse LL, Mood D, Kershaw T, Schafenacker A, Mellon S, Walker J, Galvin E, Decker V (2002) Quality of life of women with 
recurrent breast cancer and their family members. J Clin Oncol 20: 4050-4064. doi:10.1200/JCO.2002.02.054

20. Tomich PL, Helgeson VS (2004) Is finding something good in bad always good? Benefit finding among women with breast cancer. Health Psychol 23:16-23. doi:10.1037/0278-6133.23.1.16

21. Lewis FM (1996) Family home visitation study final report. National Cancer Institute, National Intitutes of Health, Bethesda

22. Kenny DA, Kashy DA, Cook WL (2006) Dyadic data analysis. Guilford: New York.

23. Kershaw T, Ellis KR, Yoon H, Schafenacker A, Katapodi M, Northouse LL (2015) The interdependence of advanced cancer patients' and their family caregivers' mental health, physical health, and self-efficacy over time. Ann Behav Med 49:901-911. doi:10. 1007/s12160-015-9743-y

24. Manne SL, Siegel SD, Heckman CJ, Kashy DA (2016) A randomized clinical trial of a supportive versus a skill-based couple-focused group intervention for breast cancer patients. J Consult Clin Psychol 84:668-681. doi:10.1037/ccp0000110

25. Manne S, Babb J, Pinover W, Horwitz E, Ebbert J (2004) Psychoeducational group intervention for wives of men with prostate cancer. Psychooncology 13:37-46. doi:10.1002/pon.724

26. Holm M, Årestedt K, Carlander I, Fürst C-J, Wengström Y, Öhlen J, Alvariza A (2016) Short-term and long-term effects of a psychoeducational group intervention for family caregivers in palliative home care-results from a randomized control trial. Psychooncology 25:795-802. doi:10.1002/pon.4004

27. Northouse LL (2005) Helping families of patients with cancer. Oncol Nurs Forum 32:743-750. doi:10.1188/05.ONF.743-750

28. Badr H, Krebs P (2013) A systematic review and meta-analysis of psychosocial interventions for couples coping with cancer. Psychooncology 22:1688-1704. doi:10.1002/pon.3200

29. Hartmann M, Bäzner E, Wild B, Eisler I, Herzog W (2010) Effects of interventions involving the family in the treatment of adult patients with chronic physical diseases: a meta-analysis. Psychother Psychosom 79:136-148. doi:10.1159/ 000286958

30. Bultz BD, Speca M, Brasher PM, Geggie PHS, Page SA (2000) A randomized controlled trial of a brief psychoeducational support group for partners of early stage breast cancer patients. Psychooncology 9:303-313. doi:10.1002/1099-1611(200007/08) 9:4<303::AID-PON462>3.0.CO;2-M

31. Cohen M, Kuten A (2006) Cognitive-behavior group intervention for relatives of cancer patients: a controlled study. J Psychosom Res 61:187-196. doi:10.1016/j.jpsychores.2005.08.014

32. Badger TA, Segrin C, Hepworth JT, Pasvogel A, Weihs K, Lopez AM (2013) Telephone-delivered health education and interpersonal counseling improve quality of life for Latinas with breast cancer and their supportive partners. Psychooncology 22:1035-1042. doi: 10.1002/pon.3101 